\title{
Molecular aspects of breast cancer resistance to drugs (Review)
}

\author{
GLORIA M. CALAF ${ }^{1,2}$, ANDREA B. ZEPEDA ${ }^{3}$, RODRIGO L. CASTILLO ${ }^{4}$, CAROLINA A. FIGUEROA $^{3}$, \\ CONSUELO ARIAS $^{3}$, ELÍAS FIGUEROA ${ }^{3,5}$ and JORGE G. FARÍAS ${ }^{3}$ \\ ${ }^{1}$ Instituto de Alta Investigación, Universidad de Tarapacá, Arica 1001236, Chile; ${ }^{2}$ Center for Radiological Research, \\ Columbia University Medical Center New York, NY 10032, USA; ${ }^{3}$ Department of Chemical Engineering, \\ Faculty of Engineering and Sciences, Universidad de la Frontera, Temuco; ${ }^{4}$ Pathophysiology Program, \\ Biomedical Sciences Institute, Faculty of Medicine, Universidad de Chile, Santiago 8380453; \\ ${ }^{5}$ School of Aquaculture, Catholic University of Temuco, Faculty of Natural Resources, Temuco, Chile
}

Received April 21, 2015; Accepted June 2, 2015

DOI: 10.3892/ijo.2015.3055

\begin{abstract}
Despite continuous advances in the knowledge of breast cancer pathophysiology, this type of neoplasia remains a leading cause of cancer-related death in women worldwide. Carcinogenesis takes a progressive course from somatic mutations, alteration of the DNA repair mechanisms, inhibition of growth suppressors, followed by cell proliferation, tissue invasion and risk of metastasis. Less than $10 \%$ of all cancers are hereditary, and in the case of breast cancer only $8 \%$, a phenomenon linked to genetic changes in BRCA1 or BRCA2. All the other cancers can be caused by an infection $(15 \%)$ or in most cases (75\%) the etiology is unknown. Patients with genetic mutations in BRCA1 or BRCA2 have $30-60 \%$ likelihood of developing a second primary breast cancer and between 11 and $45 \%$ risk of ovarian cancer, HER-2/neu is overexpressed in $\sim 30 \%$ of human breast tumors and it has a predictive role in chemotherapy and endocrine therapy.
\end{abstract}

\section{Contents}

1. Introduction

2. Risk factors

3. Prognostic and predictive factors

4. Traditional treatment using TNM staging for invasive breast cancer

5. Proposed hypotheses

\section{Introduction}

Cancer is a group of diseases characterized by the growth and uncontrolled propagation of abnormal cells. It is the end result

Correspondence to: Dr Jorge G. Farías, Department of Chemical Engineering, Faculty of Engineering and Sciences, Universidad de La Frontera, P.O. Box 54-D, Temuco, Chile

E-mail: jorge.farias@ufrontera.cl

Key words: breast cancer, multidrug resistance, cancer classifications of successive genetic changes, altering regulation processes, producing tumor cells with important advantages of survival and growth. Breast cancer is one of the most common cancers in the USA and one of the main causes of cancer-related death in women worldwide $(1,2)$. In the last 25 years in both North America and Europe, mortality from breast cancer has fallen as a result of early detection with screening program like mammography and the advent of more efficacious adjuvant systemic therapy (3). Fig. 1 summarizes the global analysis of aspects related to the treatment of breast cancer that includes a classification by using traditional and molecular methods, identification of the different prognostic factors considering the patient's individual risk. A traditional clinical form considers TNM staging system that takes into account the size of the tumor (T), lymph nodes (N) and metastasis (M) (4). TNM combinations are one of five possible stages where 0 is a carcinoma in situ and 4 is a cancer that has spread to other organs; or classifying it according to the part of the breast where the cancer originated such as ductal or lobular (5). According to histological observations it has distinguished the in situ carcinoma with ductal and lobular subtypes which constitute the majority of all breast cancers worldwide $(3,4)$, and the invasive carcinoma with tubular, ductal lobular, invasive lobular, infiltrating ductal, mucinous, medullary and infiltrating ductal subtypes (4).

A molecular form includes genetic analysis as chromosomes $6,7,21$ or molecular biotechnology, using biological markers as estrogen receptor $\left(\mathrm{ER}^{+} / \mathrm{ER}^{-}\right)$, progesterone receptor $\left(\mathrm{PR}^{+} / \mathrm{PR}^{-}\right)$, and human epidermal growth factor receptor 2 (HER-2 $/$ HER-2-) (5). In addition, other factors can be analyzed that can be originated in the cancer cell itself as mutations in p53, EGFR or influenced by microenvironment as $\mathrm{pH}$ and MMP9 $(6,7)$. According to some gene expression profile studies five different subtypes have been identified (8,9): a) luminal ${\mathrm{A}\left(\mathrm{ER}^{+} \text {and/or PR }\right.}^{+}$, HER-2-), b) luminal $\mathrm{B}\left(\mathrm{ER}^{+}\right.$and/or $\left.\mathrm{PR}^{+}, \mathrm{HER}-2^{+}\right)$, c) overexpression of HER-2 (ER-, PR', HER-2+) and d) basal (ER', PR', HER-2-, cytokeratin $5 / 6^{+}$and/or epidermal growth factor receptor ${ }^{+}$).

\section{Risk factors}

The various known risk factors associated with breast cancer can be divided into endogenous factors (age of first menstrua- 
tion, menopause, first pregnancy, lactation, number of children) and exogenous (hormone replacement therapy, contraception) (10). In recent years, metabolic risk factors such as obesity, high cholesterol and diabetes have been studied, which individually or combined have been associated with increased breast density, which is an important intermediate biomarker in breast cancer (11). However, there are also genetic risk factors characteristic of breast cancer. Analysis of mutations in genes BRCA1 and BRCA 2 in patients diagnosed with breast cancer may be an important factor in treatment decisions (12). These genes have been linked to an increase in hereditary breast cancer risk based on personal and family history (12). In fact, patients with genetic mutations in BRCA1 or BRCA2 have $30-60 \%$ likelihood of developing a second primary breast cancer and between 11 and $45 \%$ risk of ovarian cancer. Genetic variants important for evaluating an individual's risk of the disease are the protein and gene expression, microRNA, polymorphisms expression and specific epigenetic changes (13). Global hypomethylation has been observed in tumor cells together with hypermethylation in tumor suppressor genes, which inactivates certain microRNA and therefore modifies the gene expression. Specifically, hypermethylation has been reported in breast cancer in genes such as BRCA1, E-cadherin, TSM1 and the estrogen receptor in addition to global hypomethylation (14). In breast cancer there are alterations in genes involved in DNA repair and mutations in TP53 (15), and BRCA1, CDKN2A and PTEN that are hypermethylated.

\section{Prognostic and predictive factors}

In cancer research, terms such as prognostic factor and predictive factor aid in the visualization of the prognosis and treatment decision-making adapted to each patient. A prognostic factor in cancer is any measurement available at the time of the surgery that correlates the rate of overall survival or being free of the disease in the absence of systemic adjuvant therapy, i.e., the natural course of the disease. In contrast, a predictive factor is any measurement associated with the response to a certain treatment (16). In the early stages the significant prognostic factors in the case of breast cancer are age, race, tumor size, nodal status, histological grade, ER and PR expression and HER-2 status. Among these factors the invasion of the lymph nodes is the most important prognostic factor in the decision to use adjuvant therapy or not, having a direct relation with distant recurrence rate $(16,17)$. The differences perceived in the clinical outcomes are frequently correlated with race, where the mortality of Afro-American women is $77 \%$ higher than white women, possibly due to a greater prevalence of basal tumors (9). In the advanced stages of breast cancer some of the prognostic factors are the hormone receptor status of the primary tumor, metastasis in the soft and bone tissue, intervals free of relapses and good functional performance of the patient (18). In terms of the predictive factors, ER and PR expression are also considered predictive, particularly in relation to the results that could be obtained with a pharmacological treatment such as tamoxifen (16), and independent of the treatment, ER expression in tumor cells is associated with a better prognosis (19). It has also been reported that the overexpression of HER-2/neu, a protooncogene overexpressed in $\sim 30 \%$ of human breast tumors, has a predictive role in chemotherapy and endocrine therapy (20). Finally, the gene CCNA2, which belongs to a highly conservative family of cyclins and is overexpressed in several types of cancer, has been associated with resistance to tamoxifen and endocrine therapies. In fact, it has been suggested that CCNA2 is a prognostic biomarker in $\mathrm{ER}^{+}$breast cancer and is useful as a predictive factor as it serves to monitor the effectiveness of tamoxifen (21). The key driver of the proliferation of ER $\alpha(+)$ is the high expression of microRNA miR-375. Overexpression of HIF1 in ER $\alpha(+)$ cells cooperates with ER and hypoxia to promote breast cancer progression. Beta protein 1 (BP1) was found to be overexpressed in ER $\alpha$ (-) tumors and results in significantly enhanced cell proliferation and metastatic potential (22).

\section{Traditional treatment using TNM staging for invasive breast cancer}

Understanding the biology of cancer is a fundamental prerequisite for an appropriate treatment (23) since the response to cancer treatment is influenced by many factors, including the pharmacokinetics and pharmacodynamics of the drugs to be used, the tumor microenvironment, the characteristic angiogenesis and the genetic aberrations (24). According to the American Cancer Society (25), the main forms of breast cancer treatment are: surgery, radiation therapy, chemotherapy, hormone therapy, targeted therapy and bone-directed therapy.

These treatments can also be classified into local or systemic and adjuvant or neoadjuvant groups. The local therapy treats the tumor from within, without affecting the rest of the body as surgery or radiation does. Systemic therapy refers specifically to the drugs that can reach the cancer cells in any part of the body. These include chemotherapy, hormone therapy and targeted therapy, among others. Adjuvant therapy is defined as the administration of chemotherapy to kill or inhibit clinically non-detectable micrometastases after the first surgery. Neoadjuvant therapy is used in the initial stages of breast cancer before surgery where some cells can produce metastasis. The purpose of both is to destroy hidden cancer cells (26) by eradicating distant micrometastatic deposits (16). The problem with this treatment is that it carries significant health risks, and therefore the selection of patients who need it must be based on predictive factors. There are significant differences in the treatment of invasive breast cancer according to its stage. By way of example and very generally, the treatment for invasive breast cancer can be divided into early and advanced stages (26).

\section{a) Invasive breast cancer in early stages}

Surgical approach. Conservative surgery of breast and axilla, total mastectomy with axillary lymph node dissection + /- reconstruction, evaluation of whether there is lymph node involvement or not (26).

Adjuvant treatment for patients with operable breast cancer. If the patients have minimum risk and are node-negative: endocrine therapy with tamoxifen is added. If the patients have moderate risk and are node-negative: treatment with luteinizing hormone-releasing hormone (LHRH) antagonist + tamoxifen. If these patients do not respond to the treatment 
Global analysis of aspects related to the treatment of breast cancer

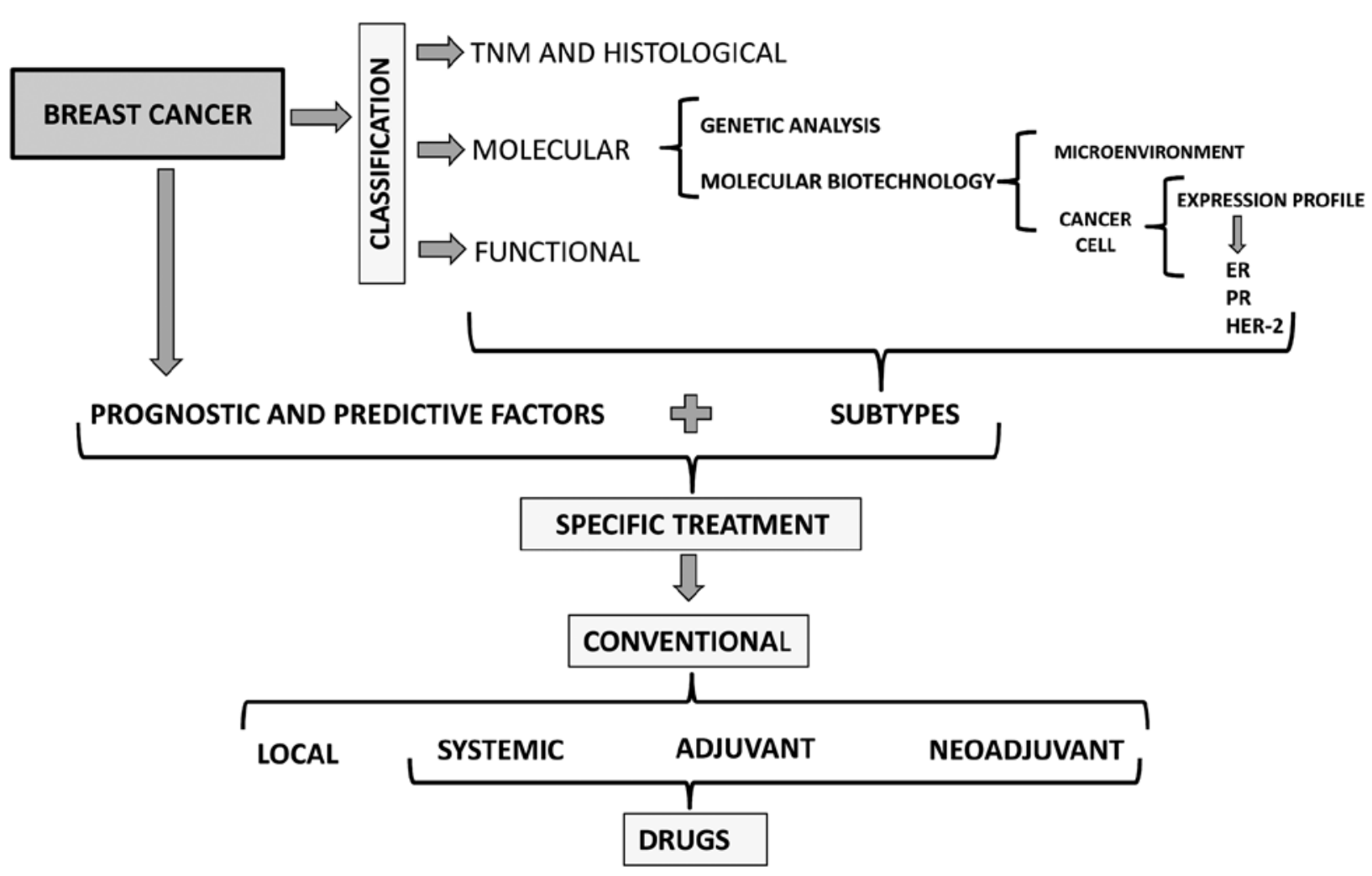

Figure 1. Global analysis of aspects related to the treatment of breast cancer. For a correct treatment decision in breast cancer an analysis must be performed that includes a classification by traditional and molecular methods, identification of the different prognostic factors and the patient's individual risk. Subtypification would facilitate treatment decisions in both conventional and alternative medicine. ER, estrogen receptor. PR, progesterone receptor. HER-2, human epidermal growth factor receptor 2.

chemotherapy is initiated. If the patients are node-positive: treatment with chemotherapy + tamoxifen (26).

\section{b) Invasive breast cancer in advanced stages}

Metastatic breast cancer. The treatment is determined according to the molecular classification, age, predictive and prognostic factors and can be first-line hormone therapy or chemotherapy according to the case, and chemotherapy as a last resort in both situations (26). Despite the advances, most clinical treatments for breast cancer including chemotherapy or hormone therapy are not very successful in eliminating metastatic cells; invasive cancer is highly lethal, in fact, patients in stage IV have a survival of 15-27 months despite the most aggressive treatment and the most effective drug use $(27,28)$. Endocrine drugs are the safest and most effective treatment in the management of hormone-sensitive breast cancer, although unfortunately tumor cells sooner or later develop a resistance to endocrine manipulation, and this has been observed in $40 \%$ of patients in advanced stages who do not respond effectively to tamoxifen $(27,29)$. This occurs because the heterogeneous population of metastatic cells generally follows a different clinical course (27) due to changes in some factors of the tumor environment (as energy metabolism) (30) or genetic or epigenetic alterations of the cancer cells (31).

Pharmacotherapy. The purpose of adjuvant and systemic therapies is to improve the disease-free survival and survival rate associated with breast cancer through local-only treatments (surgery and radiation). For many years the treatment recommendations for adjuvant therapies were based on the anatomical classification and pathological factors such as tumor size, tumor grade and lymph node status. With the development of immunohistochemistry and genomic profiling techniques, different subtypes have been classified based on various markers and more specific therapies have been developed with a greater number of clinical benefits (32). There are a large number of drugs approved by Food and Drug Administration (FDA) for breast cancer. The list contains generic or brand names, and also includes the combinations in which they are used such as lapatinib in combination with capecitabine for previously treated metastatic breast cancer that overexpresses HER-2 (33).

Classification of pharmacological agents. Chemotherapeutic agents can be classified according to many factors, including the specific phase of toxicity or the mechanism of action (34).

\section{Specific phase of toxicity}

a) Phase-specific chemotherapy. Methotrexate inhibits DNA synthesis; alkaloids inhibit the M phase of the cycle.

b) Cell cycle-specific chemotherapy. In cells that divide actively there is a dose-related plateau due to loss of sensitivity, which is offset with increased exposure time. 
c) Non-specific chemotherapy. Alkylating agents with the same independent effect on the cell cycle.

\section{Specific mechanisms}

a) Alkylating agents. They add an alkyl group, interfering mainly in DNA replication (cyclophosphamide among others).

b) Heavy metals. Platinum-based agents: carboplatin, cisplatin and oxaplatin bond mainly to DNA.

c) Antimetabolites. They simulate the structure of natural substances like vitamins, nucleosides or amino acids. They act during the $\mathrm{S}$ phase. Folic acid antagonists: methotrexate. Pyrimidine analogs inhibit synthesis of nucleic acids. e.g., fluorouracil. Purine analogs inhibit synthesis of nucleic acids. e.g., 6-mercaptopurine.

d) Cytotoxic antibiotics. They alter synthesis and function of nucleic acids. Anthracyclins intercalate into the DNA and affect DNA enzymes. e.g., doxorubicin. Actinomycin D intercalates between $\mathrm{G}$ and $\mathrm{C}$. Bleomycin: mixture of oligopeptides that fragment the DNA. Mitomycin C inhibits DNA synthesis.

e) Spindle toxins. Vinca alkaloids prevent formation of the spindle binding to the tubulin. Taxoids activate apoptotic pathways, inhibiting disassembly of microtubules, such as paclitaxel or Taxol.

f) Topoisomerase inhibitors. They alter the 3D structure of the DNA. Topoisomerase inhibitors prevent replication. e.g., camptothecin. Topoisomerase II inhibitors cause DNA fibers to rupture and prevent replication.

Since alteration of the cell cycle plays a pivotal role in the pathogenesis of cancer, having cyclins, CDKs and CDKIs (the former are positive regulators and the latter negative regulators of the cell cycle) as targets is a recurring theme in oncology (34). In 1990 taxanes (paclitaxel and docetaxel) were used as powerful and effective antitumor drugs in advanced breast cancer with a $17 \%$ reduction in the risk of recurrence although with an elevated rate of side effects (32).

Multidrug resistance. Multidrug resistance (MDR) is the greatest obstacle in the systemic treatment of breast cancer; it renders the disease uncontrollable and causes high mortality (35-39). It is therefore of the utmost importance to find drugs that can control breast cancer and offer better results. MDR can be intrinsic, where a fault is generated in the first-line therapy, which indicates there are pre-existing factors that mediate resistance in the tumor cells, or acquired, where initially the cells were sensitive to the treatment but resistance developed later on $(30,39,40)$. The latter is characterized as being a resistance parallel to several drugs with a diverse structure and function and which are made up of different cell factors and transduction signals (36). In addition, as tumors are heterogeneous, it is believed that drug resistance may originate as a positive selection of a sub-population of drugresistant cells (39). Fig. 2 shows several possible mechanisms involved in drug resistance.

a) Epithelial-mesenchymal transition (EMT). The epithelial cancer cells lose their polarity and their tight junctions, developing a fibroblast morphology associated with an increased mobility and invasiveness. Alterations in TGF $\beta$ signaling have been involved. Cancer cells with EMT and cSC characteristics also acquire resistance through EMT or ATP-binding cassette (ABC) $(29,35,39)$.

b) Epigenetic changes. Epigenetic silencing of tumor suppressor genes such as TP53 or PKD1 that can influence the tumor microenvironment and promote the progression of a breast tumor to an aggressive metastatic phenotype $(2,28,39)$.

c) miRNA. Various studies show that miRNAs are key regulators in drug resistance in breast cancer through the positive regulation of drug efflux transporters, anti-apoptotic proteins, acquisition of the EMT and the formation of CSC $(36,37)$. MicroRNAs are small non-coding RNA that silence translation through the interaction of homologous 3UTR regions in specific target RNA. Many tumors have an overall downregulation of miRNA expression (41). miRNAs could be potential biomarkers able to predict response to systemic therapy, prognosis, and guide adjustments in clinical treatment (36).

d) ABC transporters. The ATP-dependent drug efflux pumps reduce the intracellular accumulation of the drug $(30,36,39)$. The best known are the multidrug resistance protein 1 (MDR1 or P-gp), the multidrug resistance-associated protein 1 (MRP1) or the breast cancer resistance protein (BCRP) (39).

e) Alterations in the drug target. Due to mutations or changes in the expression levels (39).

f) Anti-apoptotic resistance. The cells become addicted to a moderate number of anti-apoptotic proteins for their survival. Deregulation of $\mathrm{Bcl}-2$ and PI3K/Akt signaling has been reported $(30,36,39)$.

Endocrine therapy. There are three classes of pharmacological agents used in hormone therapy to treat $\mathrm{ER}^{+}$breast cancer (42): a) selective estrogen receptor modulators (SERMs) such as tamoxifen; b) estrogen synthesis inhibitors like anastrozole, letrozole and exemestane; c) selective estrogen receptor degraders (SERDs), such as fulvestrant.

Tamoxifen has been reported as being of significant benefit to hormone-sensitive patients. It reduces the risk of recurrence after 5 years by $41 \%$ and of mortality by $34 \%$ and for decades has been the most successful treatment for $\mathrm{ER}^{+}$cancer $(32,42)$.

Endocrine therapy is considered the standard hormone therapy for patients with endocrine-sensitive tumors as defined by the ER or PR expression $(1,32)$; although the classification of breast cancer as endocrine-sensitive depends on the hormones or positive for the estrogen receptor is referred to particularly as ER $\alpha$ (19). Endocrine therapy is based on three different strategies, which include: i) ER blockade through the use of selective ER modulators (SERM) like tamoxifen; ii) reduction in estrogen levels through aromatase inhibitors (AIs); iii) induction of ER degradation by SERDs like fulvestrant $(29,42)$. ER belongs to the superfamily of nuclear receptors (29). There are two different molecular forms for the ER described, ER $\alpha$ and ER $\beta$, which are coded by different genes and their expression has a specific tissue distribution, and in some cases shared distribution $(29,42)$. ER activation can interact or activate directly or indirectly various growth factors of receptor tyrosine kinases (RTK) like HER-2, the 


\section{Mechanisms involved in drug resistance}

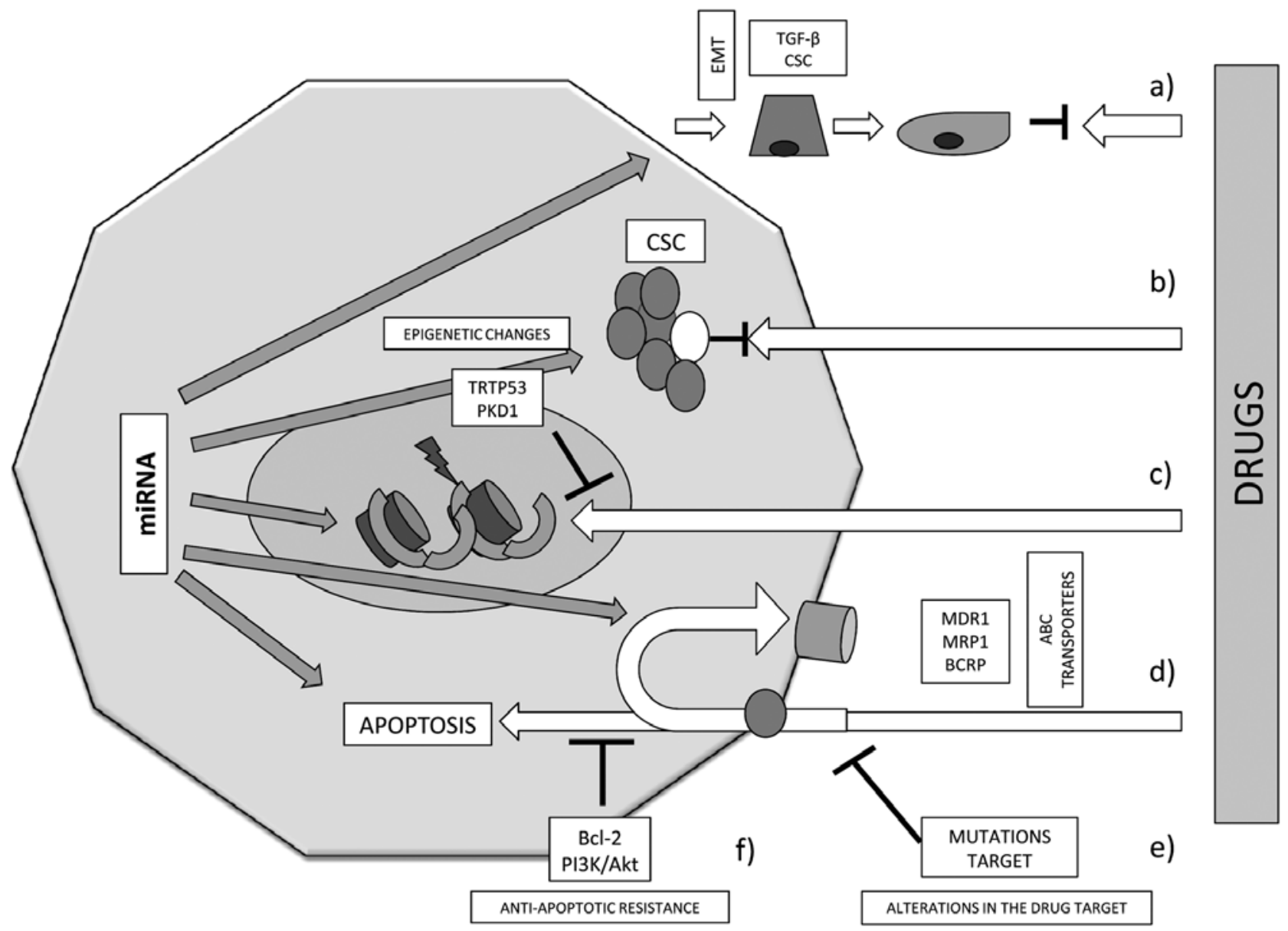

Figure 2. Mechanisms of multidrug resistance. There are several mechanisms involved in drug resistance, as anti-apoptotic resistance, epithelial-mesenchymal transition, $\mathrm{ABC}$ transporters, epigenetic changes, alterations in the drug target and miRNA (micro-ribonucleic acid). EMT, epithelial-mesenchymal transition. TGF- $\beta$, transforming growth factor $\beta$. CSC, cancer stem cells. TRTP53, telomerase reverse transcriptase p53. PKD1, polycystic kidney disease 1 gene. MDR1, multidrug resistance protein 1. MRP1, multidrug resistance-associated protein 1. BCRP, breast cancer resistance protein.miRNA, microRNA.

epidermal growth factor receptor (EGFR), and insulin-like growth factor 1 receptor (IGF1R), among others (42). ER $\alpha$ has been associated most with carcinogenesis, whereas the loss of ER $\beta$ expression has been involved in tumor progression $(19,42)$. The blocking of the signaling mediated by ER $\alpha$ is the mechanistic model for all hormonal interventions used to treat this disease (19). ER $\alpha$ (-) breast cancer remains one of the most therapy-resistant diseases, because of that differential protein expression between ER (+) and ER (-) tissues has been investigated (22). ER $\alpha$ (-) cells express lower levels of: i) superoxide dismutase, ii) RalA binding protein, iii) galectin-1, among others and expressed higher levels of Rho GDP-dissociation inhibitor $1 \alpha$. The collective role of the alterations of protein expression in ER $\alpha$ (-) cells may be to promote a more malignant phenotype than adjacent ER $\alpha(+)$ cells (22).

Mechanisms of endocrine resistance. According to authors (29) various resistance mechanisms can be found in endocrine therapy.

a) Loss of ERa expression. At both the epigenetic and transcriptional levels. In 20\% of patients there is a loss of ER (42), a phenomenon that occurs with endocrine therapy with selective endocrine receptor modulators (SERM), AIs and SERDs. b) ER $\alpha$ mutations. These occur in $<1 \%$ of patients with $\mathrm{ER}^{+}$ tumors (42). They appear mainly in endocrine therapy with SERM.

c) Loss of ER $\beta$ expression. This occurs mainly in endocrine therapy with SERM.

d) PR deficiency. This occurs mainly in endocrine therapy with SERM.

e) Alteration in the metabolism of the drug. This occurs mainly in endocrine therapy with SERM.

f) Increase in growth factor signaling. The increase in the expression of EGFR, HER-2 and IGF1R causes resistance in particular to tamoxifen, but it can also appear in therapy with SERM, AIs and SERDs.

g) Increase in ER sensitivity. This occurs in endocrine therapy with SERM, AIs and SERDs.

Monoclonal antibodies. Targeted therapy represents the greatest hope in the war against cancer and is a very important step in personalized medicine. To avoid the various toxic effects of some drugs, antibody-drug conjugates have been developed that can aim the treatment specifically at tumor cells. One of these antibodies is trastuzumab emtansine (T-DM1), which can block the extracellular part of the bond to 
the HER-2 receptor ligand, inhibiting the pathological signal of HER-2 overexpression inside the cell. Because the benefit in the clinical response rate and absolute survival is small, more effective drugs are needed that can be conjugated with T-DM1 (43). Despite an optimal local treatment, virtually all patients with invasive breast cancer have some risk of relapse. This risk varies according to several factors related to the disease, which is why all women with this type of cancer could obtain benefits from adjuvant therapy. However, all these treatments have indirect side effects and potential risks that must be considered when evaluating the need for systemic therapy (26). In fact the side effects from adjuvant chemotherapies can be fatal in $1 \%$ of patients (32) and $~ 90 \%$ of mortality in breast cancer is due to metastases resistant to adjuvant therapies (44). Some of the acute and chronic side effects associated with breast cancer treatment are cardiotoxicity, one of the most important (for treatment with anthracyclins, radiation therapy, etc.), reproductive dysfunction, pneumonitis, arm lymphedema, changes in the skin, and others $(45,46)$. Some effects of endocrine therapy are documented mainly with the use of tamoxifen and include pulmonary embolism, deep vein thrombosis and endometrial cancer. The aromatase inhibitors have been associated with an increased risk of cardiovascular disease, high cholesterol and acceleration of bone tissue loss in post-menopausal women (47).

Cancer stem cells. It is known now that cancer cell populations contain a subpopulation of self-renewing stem cells known as cancer stem cells (CSC). Unlike normal adult stem cells that remain constant in number, such cells can increase in number as tumors grow, and give rise to progeny that can be both locally invasive and colonize to distant sites known as one of the hallmarks of malignancy. Cancer begins in normal somatic cells with a mutation that is called initiation. If the mutated cell has a selective growth advantage over its normal neighbor cells due to enhanced proliferation or resistance to apoptosis, then a clone of cells carrying the same mutation will emerge. The microenvironment surrounding blood vessels is conducive to the highest rates of tumor-cell proliferation, but it can also serve as the local stem cell niche, a microenvironment formed by nerves, mesenchymal cells and molecules of extracellular matrix that regulate aspects of stem cell behavior. The insensitivity of cancer stem cells to chemotherapy and radiation treatment has suggested that anticancer drugs may not effectively inhibit such cells, thus targeting them will be important to eradicate tumors in an efficient way. The clinical relevance of targeting genes of such cells is supported by experimental and clinical studies.

It is necessary to take into account that the mesenchymal phenotype is associated with characteristics of CSC for the development of anti-CSC therapies in the future. Incorporating the concepts of CSCs and the EMT into the biology of cancer may dramatically change the paradigm of anticancer therapy. The overall goal of cancer therapy is to target only the cancer cells leaving the viable normal cells behind. Therefore CSC eradication seems possible. Thus targeting of CSCs by their specific cell surface markers seems a logical approach to target therapy than other targeting strategies would be like signaling pathways or extracellular. If we can identify different populations of cells that exhibit cell surface markers and identify them as stem cells it will be possible to evaluate the effectiveness of new targeting strategies on that population. If we can show that there are cells that have specific behaviors to decrease apoptosis and make them resistant, then we are one step further in finding treatment to destroy them. There is a critical need for more direct markers to reduce the tumor microenvironment or circulating CSC to assess the direct impact of those CSC targeted therapies.

Tumor initiating cells have been identified in a variety of cancers by sorting of subpopulations based on cell surface markers and transplantation into animal models. Human breast epithelial cells with stem cell properties have been previously characterized based on cell surface marker. These cells exist in a less differentiated state and can proliferate in suspension to form mammospheres (48). Studies highlight the need to develop new therapeutic strategies which can target cancer stem cells. Among the cell surface markers, CD44 is expressed mostly in basal-like cell lines whereas CD24 in luminal-like cell lines. Other putative markers including $\mathrm{CD} 44^{+} / \mathrm{CD} 24 \%$ Low, Her2, ALDH, CD133, ER, PR and a6-integrin etc. are also overexpressed and drive tumorigenesis in different breast cell lines (49-51). In breast cancer CD44 $/$ CD24\% low cells were identified as candidate breast cancer stem cells based on xenotransplant assays in immunodeficient mice (49). They investigated the genome-wide gene expression profiles of these cells, called, $\mathrm{CD} 44^{+}$and more differentiated luminal epithelial CD44-CD24 $4^{+}$, called, CD24+. Specifically, CD $44^{+}$cells showed a more mesenchymal stem cell-like profile enriched for genes involved in cell motility, proliferation, and angiogenesis, whereas CD24+ ${ }^{+}$cells highly expressed genes implicated in carbohydrate metabolism and RNA splicing. In general the prevalence of $\mathrm{CD} 24^{+} / \mathrm{CD} 24 /$ low cells in breast cancer may not be associated with clinical outcome but may favor distant metastasis. Authors (52) reported that circulating tumor cells and biomarker can be considered for personalized targeted treatments for metastatic breast cancer.

A functional classification of breast cancer has proposed two hypotheses based on either breast cancer heterogeneity that arise from distinct mammary stem/progenitor cells at various levels or as a result of a single mammary stem/progenitor cell being affected by various oncogenes which give rise to various types of cancer (4). This has enabled the distinction of different molecular subtypes in breast cancer (25), and their identification make it possible to select a specific treatment strategy and to better predict the prognosis (6). Approximately $70 \%$ of all breast cancers diagnosed in the USA are $\mathrm{ER}^{+}(22,36)$. Currently one of the major efforts in integrative genomics related to cancer research is The Cancer Genome Atlas (TCGA), in which the genotype, epigenome, transcriptome, proteome, morphology and clinical records of hundreds of patients are available for each type of cancer including breast cancer (53).

Advances in research related to the classification of cancer have opened up new doors in terms of treatment. In light of tumor drug resistance cells, new therapeutic approaches are needed that can improve prognosis. Since cancer is a prevalent cause of mortality in adults, basic research and clinical studies are required to analyze the risks and benefits of these alternative therapies, and their connection to drug resistance. Such approaches would be important to implement since some 
patients, while their survival increases, are exposed to other pathologies and risks such as chronic diseases, among which cancer is emphasized. It is thought that one of the ways to prevent or overcome endocrine resistance is the combination of different targets in the therapy that effectively block both ER and RTK-dependent signaling (42), among other molecular mechanisms.

\section{Other therapies}

Antioxidants. Oxidative stress has an important role in tumor growth and in conjunction with inflammatory processes is directly related to cancer development, progression and metastasis (54). Antioxidants have been studied in patients with cancer in the first place as potential anticancer agents that could improve prognosis, and second as reducers of the oxidative damage caused by chemotherapy and radiation therapy (55). Despite their potential use in therapies and preventive applications, their effects have been controversial (54). In fact, it has been reported that some supplements with vitamin A or E could even increase mortality significantly reducing in turn the effect of the conventional therapy $(54,55)$. Conversely, there has been a statistically significant association between the dietary supplement vitamin $\mathrm{C}$ and a reduction in the risk and total and specific mortality in patients with breast cancer (56).

Another substance is the curcumin mayor component of the spice turmeric (Curcuma longa) and for several decades has proven to be a powerful anti-inflammatory agent and to have a great therapeutic potential against several types of cancer, blocking the transformation, proliferation and invasion of tumor cells (57-59). Curcumin suppresses multiple signaling pathways (58), such as caspase activation, induction of cell death receptors, aggregation of Fas receptors, induction of p53, and p21 pathways (57), release of apoptosis-inducing factor $(57,60)$, regulation of the cell cycle $(58,61)$, inhibition of PI3K-AKT activation, mTOR inhibition, downregulation of androgen receptors (57), inhibition of AMP-activated protein kinase, inhibition of COX2 and LOX 5, inhibition of STAT3 activation (57), c-Jun kinase activation, induction of DNA fragmentation, depletion of intracellular $\mathrm{Ca}^{2}$, mitochondrial activation, direct damage to the DNA, anti-apoptotic protein suppression, autophagy, antioxidant mechanisms, proteasome activation, $N F-\kappa \beta$ inhibition, among others $(58,61)$. Thus, many studies have focused on improving its clinical effectiveness by modifying its delivery system through nanotechnology $(62,63)$.

\section{Proposed hypotheses}

Several hypotheses have been proposed that describe the events in those cancers where the etiology has been defined. One hypothesis is the classic model of breast cancer progression of the ductal type that proposes that neoplastic evolution is initiated in normal epithelium, progresses to flat epithelial atypical (FEA), advances to atypical ductal hyperplasia (ADH), evolves to ductal carcinoma in situ and culminates as invasive ductal carcinoma. This observation is based on immunohistochemical, genomic and transcriptomic data (3) and the alternative model, which was mostly based on epidemiological and morphological observations, proposes usual ductal hyperplasia (UDH) instead of FEA as the direct precursor to ADH (3).

Another model that explains the progression of cancer is the cancer stem cell (CSC) model which postulates that only stem and progenitor cells can initiate and maintain tumor progression. It has been proposed that such cells are candidates for accumulating genetic and epigenetic modifications as a result in deregulation of normal self-renewal, leading to the development of a CSC (3). It has also been reported that the deregulation of the normal dynamics of the extracellular matrix (ECM) is a frequent characteristic in the origin and progression of cancer where stromal cells, fibroblasts are associated with cancer and immune cells. The alteration of the ECM changes promote the formation of a tumorigenic microenvironment, stimulating angiogenesis through angiogenic factors like VEGF, which determines the beginning of the vascular branches and facilitates metastasis of tumor cells, with infiltration of immune cells and progression of the cancer (64). A sequence of processes, including transition of normal to cancerous cells and later events such as proliferation, migration, invasion, mutations, and angiogenesis have been reported (65). All these steps accompanied by pathogenic stimuli, chronic inflammation, fibrosis, formation of precancerous niches, help the cells escape chronic stress. Most breast cancers begin in the highly polarized luminal epithelial cells of the breast ducts, polarity that is lost as the cancer progresses. Probably influenced by transforming growth factor $\beta$ receptor type III (TGF- $\beta$ receptor type III), which promotes epithelial-mesenchymal transition (EMT). Such disruption affects all the above processes (66). It has been reported that epigenetic changes can contribute to the development of cancer through the inactivity of tumor suppressor genes and through the activation of oncogenes; both effects occur as a result of changes in the chromatin configuration, which alters the accessibility of the transcription factors with consequences in the gene expression. The genome of breast cancer usually contains hundreds of genetic changes, of which a small subgroup can direct the course of the disease. In breast cancer the tumor suppressor gene promoters BRCA1, CDKN2A and PTEN are hypermethylated; there are alterations in genes involved in DNA repair and mutations in TP53 (15).

\section{Acknowledgements}

The technical assistance of Angela Gonzalez, Leodán A. Crispin is greatly appreciated. The authors are sincerely thankful for the support provided by DIUFRO grant DI15-2018 (JGF), CONICYT. Scholarships for PhD in Chile (C.A., A.B.Z. and C.A.F.), FONDECYT grant no. 1120006 (G.M.C. and J.G.F.) and Convenio de Desempeño MINEDUC, Universidad de Tarapacá (GMC).

\section{References}

1. Pritchard JE, Dillon PM, Conaway MR, Silva CM and Parsons SJ: A mechanistic study of the effect of doxorubicin/adriamycin on the estrogen response in a breast cancer model. Oncology 83: 305-320, 2012.

2. Borges S, Döppler H, Perez EA, Andorfer CA, Sun Z, Anastasiadis PZ, Thompson E, Geiger XJ and Storz P: Pharmacologic reversion of epigenetic silencing of the PRKD1 promoter blocks breast tumor cell invasion and metastasis. Breast Cancer Res 15: R66, 2013. 
3. Bombonati A and Sgroi DC: The molecular pathology of breast cancer progression. J Pathol 223: 307-317, 2011.

4. Zeng T and Liu J: Mixture classification model based on clinical markers for breast cancer prognosis. Artif Intell Med 48: 129-137, 2010.

5. Vuong D, Simpson PT, Green B, Cummings MC and Lakhani SR: Molecular classification of breast cancer. Virchows Arch 465 1-14, 2014.

6. Dumay A, Feugeas JP, Wittmer E, Lehmann-Che J, Bertheau P, Espié M, Plassa LF, Cottu P, Marty M, André F, et al: Distinct tumor protein p53 mutants in breast cancer subgroups. Int J Cancer 132: 1227-1231, 2013.

7. Zhang X, Jin G, Li J and Zhang L: Association between four MMP-9 polymorphisms and breast cancer risk: A meta-analysis. Med Sci Monit 21: 1115-1123, 2015

8. Turkoz FP, Solak M, Petekkaya I, Keskin O, Kertmen N, Sarici F, Arik Z, Babacan T, Ozisik Y and Altundag K: Association between common risk factors and molecular subtypes in breast cancer patients. Breast 22: 344-350, 2013.

9. Carey LA, Perou CM, Livasy CA, Dressler LG, Cowan D, Conway K, Karaca G, Troester MA, Tse CK, Edmiston S, et al Race, breast cancer subtypes, and survival in the Carolina Breast Cancer Study. JAMA 295: 2492-2502, 2006.

10. Arveux P and Bertaut A: Epidemiology of breast cancer. Rev Prat 63: 1362-1366, 2013 (In French).

11. Tehranifar P, Reynolds D, Fan X, Boden-Albala B, Engmann NJ Flom JD and Terry MB: Multiple metabolic risk factors and mammographic breast density. Ann Epidemiol 24: 479-483, 2014.

12. Vadaparampil ST, Quinn GP, Miree CA, Brzosowicz J, Carter B and Laronga $\mathrm{C}$ : Recall of and reactions to a surgeon referral letter for BRCA genetic counseling among high-risk breast cancer patients. Ann Surg Oncol 16: 1973-1981, 2009.

13. Li N, Zhou P, Zheng J, Deng J, Wu H, Li W, Li F, Li H, Lu J, Zhou Y, et al: A polymorphism rs $12325489 \mathrm{C}>\mathrm{T}$ in the lincRNAENST00000515084 exon was found to modulate breast cancer risk via GWAS-based association analyses. PLoS One 9: e98251, 2014.

14. Esteller M: Epigenetics in cancer. N Engl J Med 358: 1148-1159, 2008

15. Stefansson OA and Esteller M: Epigenetic modifications in breast cancer and their role in personalized medicine. Am J Pathol 183 $1052-1063,2013$

16. Cianfrocca M and Goldstein LJ: Prognostic and predictive factors in early-stage breast cancer. Oncologist 9: 606-616, 2004

17. Castellano I, Chiusa L, Vandone AM, Beatrice S, Goia M, Donadio M, Arisio R, Muscarà F, Durando A, Viale G, et al: A simple and reproducible prognostic index in luminal ER-positive breast cancers. Ann Oncol 24: 2292-2297, 2013.

18. Chang J, Clark GM, Allred DC, Mohsin S, Chamness G and Elledge RM: Survival of patients with metastatic breast carcinoma: Importance of prognostic markers of the primary tumor. Cancer 97: 545-553, 2003.

19. Higa GM and Fell RG: Sex hormone receptor repertoire in breast cancer. Int J Breast Cancer 2013: 284036, 2013.

20. Borg A, Tandon AK, Sigurdsson H, Clark GM, Fernö M, Fuqua SA, Killander D and McGuire WL: HER-2/neu amplification predicts poor survival in node-positive breast cancer. Cancer Res 50: 4332-4337, 1990

21. Gao T, Han Y, Yu L, Ao S, Li Z and Ji J: CCNA2 is a prognostic biomarker for $\mathrm{ER}^{+}$breast cancer and tamoxifen resistance. PLoS One 9: e91771, 2014

22. Sahab ZJ, Man YG, Byers SW and Sang QX: Putative biomarkers and targets of estrogen receptor negative human breast cancer. Int J Mol Sci 12: 4504-4521, 2011.

23. Akin S, Babacan T, Sarici F and Altundag K: A novel targeted therapy in breast cancer: Cyclin dependent kinase inhibitors. J BUON 19: 42-46, 2014

24. Muellner MK, Uras IZ, Gapp BV, Kerzendorfer C, Smida M, Lechtermann H, Craig-Mueller N, Colinge J, Duernberger G and Nijman SM: A chemical-genetic screen reveals a mechanism of resistance to PI3K inhibitors in cancer. Nat Chem Biol 7: 787-793, 2011.

25. American Cancer Society 2013: Breast Cancer Facts and Figures 2013-2014. Atlanta: American Cancer Society. 2013. http://www. cancer.org/acs/groups/content/@research/documents/document/ acspc-042725.pdf

26. World Health Organization (WHO): Guidelines for management of breast cancer. 2006. http://applications.emro.who.int/dsaf/ dsa697.pdf.
27. Dawood S, Broglio K, Gonzalez-Angulo AM, Buzdar AU, Hortobagyi GN and Giordano SH: Trends in survival over the past two decades among white and black patients with newly diagnosed stage IV breast cancer. J Clin Oncol 26: 4891-4898, 2008.

28. Borges S, Döppler HR and Storz P: A combination treatment with DNA methyltransferase inhibitors and suramin decreases invasiveness of breast cancer cells. Breast Cancer Res Treat 144: 79-91, 2014

29. Zilli M, Grassadonia A, Tinari N, Di Giacobbe A, Gildetti S, Giampietro J, Natoli C and Iacobelli S; Consorzio Interuniversitario Nazionale per la Bio-Oncologia (CINBO) Molecular mechanisms of endocrine resistance and their implication in the therapy of breast cancer. Biochim Biophys Acta 1795: 62-81, 2009

30. Tavares-Valente D, Baltazar F, Moreira R and Queirós O: Cancer cell bioenergetics and $\mathrm{pH}$ regulation influence breast cancer cell resistance to paclitaxel and doxorubicin. J Bioenerg Biomembr 45: 467-475, 2013

31. Gottesman MM: Mechanisms of cancer drug resistance. Annu Rev Med 53: 615-627, 2002

32. Hernandez-Aya LF and Gonzalez-Angulo AM: Adjuvant systemic therapies in breast cancer. Surg Clin North Am 93 473-491, 2013

33. Ryan Q, Ibrahim A, Cohen MH, Johnson J, Ko CW, Sridhara R, Justice R and Pazdur R: FDA drug approval summary: Lapatinib in combination with capecitabine for previously treated metastatic breast cancer that overexpresses HER-2. Oncologist 13 1114-1119, 2008

34. Payne S and Miles D: Mechanisms of anticancer drugs. ScottBrown's Otorhinolaryngology. Head Neck Surg 7: 34-46, 2008.

35. Piva M, Domenici G, Iriondo O, Rábano M, Simões BM, Comaills V, Barredo I, López-Ruiz JA, Zabalza I, Kypta R, et al: Sox 2 promotes tamoxifen resistance in breast cancer cells. EMBO Mol Med 6: 66-79, 2014.

36. Tian W, Chen J, He H and Deng Y: MicroRNAs and drug resistance of breast cancer: Basic evidence and clinical applications. Clin Transl Oncol 15: 335-342, 2013.

37. Chen J, Tian W, Cai H, He H and Deng Y: Down-regulation of microRNA-200c is associated with drug resistance in human breast cancer. Med Oncol 29: 2527-2534, 2012.

38. Chen SJ, Luan J, Zhang HS, Ruan CP, Xu XY, Li QQ and Wang NH: EGFR-mediated G1/S transition contributes to the multidrug resistance in breast cancer cells. Mol Biol Rep 39: 5465-5471, 2012

39. Holohan C, Van Schaeybroeck S, Longley DB and Johnston PG: Cancer drug resistance: An evolving paradigm. Nat Rev Cancer 13: 714-726, 2013.

40. Coley HM: Mechanisms and strategies to overcome chemotherapy resistance in metastatic breast cancer. Cancer Treat Rev 34: 378-390, 2008

41. van Kouwenhove M, Kedde M and Agami R: MicroRNA regulation by RNA-binding proteins and its implications for cancer. Nat Rev Cancer 11: 644-656, 2011.

42. Giuliano M, Schifp R, Osborne CK and Trivedi MV: Biological mechanisms and clinical implications of endocrine resistance in breast cancer. Breast 20 (Suppl 3): S42-S49, 2011.

43. Lianos GD, Vlachos K, Zoras O, Katsios C, Cho WC and Roukos DH: Potential of antibody-drug conjugates and novel therapeutics in breast cancer management. Onco Targets Ther 7: 491-500, 2014.

44. Ren Z, Li Y, Hameed O, Siegal GP and Wei S: Prognostic factors in patients with metastatic breast cancer at the time of diagnosis. Pathol Res Pract 210: 301-306, 2014.

45. Agrawal S: Late effects of cancer treatment in breast cancer survivors. South Asian J Cancer 3: 112-115, 2014.

46. Schmitz KH, Prosnitz RG, Schwartz AL and Carver JR: Prospective surveillance and management of cardiac toxicity and health in breast cancer survivors. Cancer 118 (Suppl): S2270-S2276, 2012

47. Rao RD and Cobleigh MA: Adjuvant endocrine therapy for breast cancer. Oncology (Williston Park) 26 (6): 541-7, 550, 552 passim, 2012

48. Dontu G, El-Ashry D and Wicha MS: Breast cancer, stem/ progenitor cells and the estrogen receptor. Trends Endocrinol Metab 15: 193-197, 2004.

49. Al-Hajj M, Wicha MS, Benito-Hernandez A, Morrison SJ and Clarke MF: Prospective identification of tumorigenic breast cancer cells. Proc Natl Acad Sci USA 100: 3983-3988, 2003 
50. Ginestier C, Hur MH, Charafe-Jauffret E, Monville F, Dutcher J, Brown M, Jacquemier J, Viens P, Kleer CG, Liu S, et al: ALDH1 is a marker of normal and malignant human mammary stem cells and a predictor of poor clinical outcome. Cell Stem Cell 1: 555-567, 2007.

51. Wright MH, Calcagno AM, Salcido CD, Carlson MD, Ambudkar SV and Varticovski L: Brcal breast tumors contain distinct $\mathrm{CD} 44^{+} / \mathrm{CD} 24^{-}$and $\mathrm{CD} 133^{+}$cells with cancer stem cell characteristics. Breast Cancer Res 10: R10, 2008.

52. Reuben JM, Lee BN, Li C, Gao H, Broglio KR, Valero V, Jackson SA, Ueno NT, Krishnamurthy S, Hortobagyi GN, et al: Circulating tumor cells and biomarkers: Implications for personalized targeted treatments for metastatic breast cancer. Breast J 16: 327-330, 2010.

53. Wang C, Machiraju R and Huang K: Breast cancer patient stratification using a molecular regularized consensus clustering method. Methods 67: 304-312, 2014.

54. Crawford S: Anti-inflammatory/antioxidant use in long-term maintenance cancer therapy: A new therapeutic approach to disease progression and recurrence. Ther Adv Med Oncol 6: 52-68, 2014.

55. Harvie M: Nutritional supplements and cancer: potential benefits and proven harms. Am Soc Clin Oncol Educ Book. 2014; 34:e478-86. doi: 10.14694/EdBook AM.2014.34.e478.

56. Harris HR, Orsini N and Wolk A: Vitamin C and survival among women with breast cancer: A meta-analysis. Eur J Cancer 50: 1223-1231, 2014.

57. Shishodia S, Chaturvedi MM and Aggarwal BB: Role of curcumin in cancer therapy. Curr Probl Cancer 31: 243-305, 2007.
58. Ravindran J, Prasad S and Aggarwal BB: Curcumin and cancer cells: How many ways can curry kill tumor cells selectively? AAPS J 11: 495-510, 2009.

59. Hasima $\mathrm{N}$ and Aggarwal BB: Targeting proteasomal pathways by dietary curcumin for cancer prevention and treatment. Curr Med Chem 21: 1583-1594, 2014.

60. Karunagaran D, Rashmi R and Kumar TR: Induction of apoptosis by curcumin and its implications for cancer therapy. Curr Cancer Drug Targets 5: 117-129, 2005.

61. Calaf GM, Echiburú-Chau C, Wen G, Balajee AS and Roy D: Effect of curcumin on irradiated and estrogen-transformed human breast cell lines. Int J Oncol 40: 436-442, 2012.

62. Cridge BJ, Larsen L and Rosengren RJ: Curcumin and its derivatives in breast cancer: Current developments and potential for the treatment of drug-resistant cancers. Oncol Discov 1: 6, 2013.

63. Terlikowska K, Witkowska A and Terlikowski S: Curcumin in chemoprevention of breast cancer. Postepy Hig Med Dosw (Online) 68: 571-578, 2014 (In Polish)

64. Lu P, Weaver VM and Werb Z: The extracellular matrix: A dynamic niche in cancer progression. J Cell Biol 196: 395-406, 2012.

65. Brücher BL and Jamall IS: Epistemology of the origin of cancer: A new paradigm. BMC Cancer 14: 331, 2014.

66. Meyer AE, Gatza CE, How T, Starr M, Nixon AB and Blobe GC: The role of TGF- $\beta$ receptor III localization in polarity and breast cancer progression. Mol Biol Cell 25: 2291-2304, 2014. 